Discrete Comput Geom 35:437-455 (2006)

DOI: $10.1007 / \mathrm{s} 00454-005-1190-2$

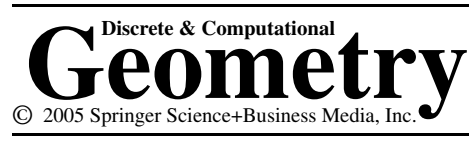

\title{
The Stirling Polynomial of a Simplicial Complex
}

\author{
Gábor Hetyei* \\ Department of Mathematics and Statistics, \\ University of North Carolina at Charlotte, \\ Charlotte, NC 28223, USA \\ ghetyei@uncc.edu
}

\begin{abstract}
We introduce a new encoding of the face numbers of a simplicial complex, its Stirling polynomial, that has a simple expression obtained by multiplying each face number with an appropriate generalized binomial coefficient. We prove that the face numbers of the barycentric subdivision of the free join of two $C W$-complexes may be found by multiplying the Stirling polynomials of the barycentric subdivisions of the original complexes. We show that the Stirling polynomial of the order complex of any simplicial poset and of certain graded planar posets has non-negative coefficients. By calculating the Stirling polynomial of the order complex of the $r$-cubical lattice of rank $n+1$ in two ways, we provide a combinatorial proof for the following identity of Bernoulli polynomials:
\end{abstract}

$$
1+r^{n} \sum_{k=1}^{n} \frac{\left(\begin{array}{c}
n \\
k-1
\end{array}\right)}{k} \cdot\left(B_{k}\left(x+\frac{1}{r}\right)-B_{k}\left(\frac{1}{r}\right)\right)=(r x+1)^{n} .
$$

Finally we observe that the Stirling polynomials of simplicial complexes associated to the cladistic characters defined by McMorris and Zaslavsky [21] are equal, up to a shift, to the Stirling polynomials defined by Gessel and Stanley [14].

\section{Introduction}

Relations between the face numbers of a cell complex are often verified using polynomial substitutions into the polynomial obtained by multiplying each face number with the appropriate power of a variable. A prototypical example of this tradition is the use of the $h$-vector of a simplicial complex [24, Chapter II, Section 2], which enables the most compact expression of the famous Upper Bound Theorem [24, Chapter II, Section 3], limiting the face numbers of a simplicial sphere of fixed dimension and with a

* On leave from the Rényi Mathematical Institute of the Hungarian Academy of Sciences. 
fixed number of vertices, as well as the Dehn-Sommerville equations [24, Chapter II, Section 6].

In this paper we suggest another equivalent encoding of the face numbers $f_{j-1}$ $(j=0, \ldots, d)$, the Stirling polynomial of a simplicial complex, given by $\sum_{k=0}^{d} s_{k} \cdot x^{k}:=$ $\sum_{j=1}^{d} f_{j-1}\left(\begin{array}{l}x \\ j\end{array}\right)$. (The terminology reflects the implied role of the Stirling numbers.) This polynomial should not be confused with the Stirling polynomials defined by Gessel and Stanley [14], although in Section 6 we show that the Stirling polynomials of certain simplicial complexes arising in association with the work of McMorris and Zaslavsky [21] on cladistic characters are the Gessel-Stanley Stirling polynomials (shifted by one).

The original motivation to study our Stirling polynomial is that, in terms of this invariant, the effect of taking the diamond product of two graded posets on the face numbers of their order complexes may be expressed as a simple polynomial multiplication. (This is shown in Section 3.) It was already known by Kalai [17] that the diamond product of the face lattices of two polytopes is the face lattice of their Cartesian product. Ehrenborg and Readdy [10] were the first to describe the effect of taking a diamond product in terms of the $c d$-index. It was recently observed by the present author [15] that the dual of this operation describes the effect of taking the free join of two $C W$-spheres on their face posets. Since the free join of two spheres is a sphere and, by a famous conjecture of Stanley [23, Conjecture 2.1], $C W$-spheres should have a positive $c d$-index, taking the dual diamond product should preserve the positivity of the $c d$-index in this case. It was shown by Ehrenborg and Fox [8] that the diamond product (and thus also its dual) of Eulerian posets always preserves the non-negativity of the $c d$-index of its arguments, independently of the original face structures. Unfortunately the explicit formulas they derive seem to be difficult to use in concrete calculations.

As a first step to a more explicit understanding of the effect of the diamond product on the flag numbers one may want to return to studying the face numbers of the order complexes involved, and that is where the Stirling polynomial of the order complex (which we refer to as the Stirling polynomial or Stirling vector of the graded poset) may be helpful. Since the original context is a search for positivity results on flag invariants, the question naturally arises: Which graded partially ordered sets have a non-negative Stirling vector? We find some surprising partial answers to this question.

The first surprise is that for an arbitrary graded simplicial poset (independently of the underlying topological structure), the Stirling polynomial has non-negative coefficients: in fact, it simply encodes the face numbers of the underlying $C W$-complex. There is also a reasonably large class of graded planar posets whose Stirling polynomials have non-negative coefficients. It was first observed by the present author and Billera [4] that the most natural enumeration of the facets in the order complex of a graded planar poset is analogous in many ways to a shelling. Surprisingly, in the study of this invariant inspired by a truly geometric operation we find a familiar "shelling-like" argument in the world of planar analogues, while in the "truly geometric sense" the underlying topology seems to play little role. (Both non-negativity results are shown in Section 4.) It turns out that the face lattice of every three-dimensional polytope has a Stirling polynomial with non-negative coefficients, and it is not yet possible to find counterexamples among fourdimensional polytopes. The fact that one needs to invoke some of the latest findings on the flag numbers of 4-polytopes to find a counterexample to Stirling positivity might indicate that the class of polytopes whose face lattice has a non-negative Stirling vector might be 
a large and interesting one. In fact, this class is shown to contain all simplicial and simple polytopes and to be closed under taking the dual or the Cartesian product of polytopes.

Although even some four-dimensional cubical polytopes occur among the counterexamples to the non-negativity of all coefficients of the Stirling polynomial, further study of the Stirling polynomial of cubical posets, and graded posets whose proper intervals $[\widehat{0}, x]$ are $r$-cubical lattices, seems to be worthwhile, since they have a compact expression in terms of the mysterious Bernoulli polynomials. A deeper understanding of the properties of the Stirling polynomial and the structure of such posets might lead to combinatorial proofs of identities for the Bernoulli polynomials. As a first example, in Section 5 we derive such an identity by calculating the Stirling polynomial of the $r$-cubical lattice in two different ways. We hope that many more example connecting the study of Stirling polynomials to that of of Bernoulli polynomials will be found in the future, and in the concluding remarks we indicate the reason why this may be the case not only for order complexes of other posets.

\section{Preliminaries}

A simplicial complex $\triangle$ is a family of subsets of a finite vertex set $V$ such that $\{v\} \in \Delta$ for all $v \in V$ and $\triangle$ is closed under inclusion. The elements of $\Delta$ are called faces. The dimension of a face $\sigma$ is $|\sigma|-1$, and the number of faces of dimension $j$ is denoted by $f_{j}$. The dimension of the complex $\Delta$ is the maximum dimension of its faces. For a $(d-1)$-dimensional simplicial complex, the vector $\left(f_{-1}, f_{0}, \ldots, f_{d-1}\right)$ is the $f$-vector of $\triangle$.

In this paper we focus on simplicial complexes that arise as order complexes of graded partially ordered sets. Given any poset $P$, its order complex $\Delta(P)$ is the simplicial complex whose vertices are the elements of $P$, and whose faces are the chains in $P$. A poset is graded if it has a unique minimum element $\widehat{0}$, a unique maximum element $\widehat{1}$, and a rank function $\rho$. For a graded poset of rank $n+1$, the order complex $\Delta(P \backslash\{\widehat{0}, \widehat{1}\})$ is completely balanced, i.e., its vertices may be colored with $\operatorname{dim}(\Delta(P \backslash\{\widehat{0}, \widehat{1}\}))+1=n$ colors in such a way that no two vertices of the same color belong to the same face. We color each vertex with its rank for this purpose. The number of faces whose vertices are colored with the set $S \subseteq\{1, \ldots, n\}$ is denoted by $f_{S}$, the vector $\left(f_{S}: S \subseteq\{1, \ldots, n)\right.$ is called the flag $f$-vector of the poset.

A graded poset is Eulerian if for all $x, y \in P$ satisfying $x<y$ we have $\sum_{x \leq z \leq y}(-1)^{\rho(z)}$ $=0$. All linear relations holding for the flag $f$-vector of an arbitrary Eulerian poset of rank $n+1$ were determined by Bayer and Billera [2].

Theorem 1.1 (Bayer and Billera). For every Eulerian poset of rank $n+1$, every subset $S \subseteq[1, n]$, and every maximal interval $[i, \ell]$ of $[1, n] \backslash S$,

$$
\left((-1)^{i-1}+(-1)^{\ell+1}\right) f_{S}(P)+\sum_{j=i}^{\ell}(-1)^{j} f_{S \cup\{j\}}(P)=0 .
$$

Furthermore, every linear equality holding for the flag vector of all Eulerian posets of rank $n+1$ is a consequence of these equations. 
These linear relations were rephrased by J. Fine (see [3]) by stating the existence of the $c d$-index (which will not be defined nor used in this paper). Fine also conjectured that the $c d$-index of the face poset of a polytope has non-negative coefficients, this result was shown by Stanley [23, Corollary 2.2]. Stanley proves this result by introducing the notion of spherical shellings for spherical $C W$-complexes, and showing that the boundary of a polytope is spherically shellable. Stanley also has a conjecture [23, Conjecture 2.1] that all Gorenstein* posets have a non-negative $c d$-index. Karu recently posted a proof of this conjecture on arXiv [18].

The poset-operation that inspired the present work is the diamond product $P \diamond Q=$ $\left(P \backslash\left\{\widehat{0}_{P}\right\}\right) \times\left(Q \backslash\left\{\widehat{0}_{Q}\right\}\right) \cup\{\widehat{0}\}$ of two graded posets $P$ and $Q$. Here $P \times Q$ denotes the Cartesian product $\{(p, q): p \in P, q \in Q\}$ with the partial order $(p, q) \leq\left(p^{\prime}, q^{\prime}\right)$ if and only if $p \leq_{P} p^{\prime}$ and $q \leq_{Q} q^{\prime}$. If both $P$ and $Q$ are Eulerian posets, so is their diamond product. The effect of this product on the $c d$-index of Eulerian posets was first studied by Ehrenborg and Readdy [10]. Ehrenborg and Fox have shown [8] that $P \diamond Q$ has a non-negative $c d$-index, whenever the same holds for $P$ and $Q$. As observed by the present author [15, Corollary 1.3], taking the free join of two $C W$-spheres $\Omega$ and $\Omega^{\prime}$ induces taking the dual diamond product at the level of face posets (where all face posets are augmented in question with a maximum element $\widehat{1}$ ). For a definition of the free join of two $C W$-complexes see Chapter 10, Section 2, of [20]; this operation is different from the free join of polytopes introduced in [17]. The term "dual diamond product" to indicate $P \diamond^{*} Q=\left(P \backslash\left\{\widehat{1}_{P}\right\}\right) \times\left(Q \backslash\left\{\widehat{1}_{Q}\right\}\right) \cup\left\{\widehat{1}_{\}}\right\}$was first used by Ehrenborg and Readdy [12].

The proof of the Ehrenborg-Fox result (which is directly applicable to the dual diamond product as well) relies on presenting some explicit formulas connecting the $c d$ indices (and thus flag $f$-vectors) of the posets involved. It seems very hard to use these formulas in explicit computations, and it seems equally hard to find simpler formulas connecting the flag $f$-vectors. The present work focuses on an invariant that makes at least the calculation of the $f$-vector of a diamond product easier (see Section 3 ).

\section{The Stirling Vector of a Simplicial Complex}

Definition 2.1. We define the Stirling polynomial of a $(d-1)$-dimensional simplicial complex in terms of its $f$-vector $\left(f_{-1}, \ldots, f_{d-1}\right)$ by the formula

$$
\sum_{k=0}^{d} s_{k} \cdot x^{k}=\sum_{j=0}^{d} f_{j-1} \cdot\left(\begin{array}{l}
x \\
j
\end{array}\right) .
$$

We refer to the vector $\left(s_{0}, \ldots, s_{d}\right)$ as the Stirling vector of the simplicial complex.

As usual, the generalized binomial coefficient $\left(\begin{array}{l}x \\ j\end{array}\right)$ stands for the polynomial

$$
\left(\begin{array}{l}
x \\
j
\end{array}\right)=\frac{(x)_{j}}{j !}=\frac{x(x-1) \cdots(x-j+1)}{j !} .
$$

The term Stirling vector is motivated by the fact that the $f$-vector may be expressed in terms of the Stirling vector using Stirling numbers of the first kind. In fact, using the 
well-known formula

$$
(x)_{n}=\sum_{k=0}^{n} s(n, k) \cdot x^{k}
$$

we may write

$$
s_{k}=\sum_{j=k}^{d} f_{j-1} \cdot \frac{s(j, k)}{j !} .
$$

Conversely, as a consequence of the well-known equation

$$
x^{n}=\sum_{k=0}^{n} S(n, k) \cdot(x)_{k}
$$

we may write

$$
f_{j-1}=j ! \sum_{k=j}^{d} S(k, j) \cdot s_{k} .
$$

Here the numbers $S(n, k)$ are the Stirling numbers of the second kind. In other words, the Stirling vector, just like the $h$-vector (see Chapter II, Section 2, of [24]), is an equivalent encoding of the $f$-vector. In analogy to the $h$-vector, the face numbers are non-negative combinations of the Stirling vector entries, thus bounds on the entries of the Stirling vector imply bounds on the entries of the $f$-vector.

\section{Face Numbers in a (Dual) Diamond Product of Graded Posets}

In this section we describe the effect of taking the diamond product of two graded posets on the face numbers of their order complexes. It should be noted that the order complex $\triangle(P \backslash\{\widehat{0}, \widehat{1}\})$ of a graded poset $P$ is identifiable with the order complex $\Delta\left(P^{*} \backslash\{\widehat{0}, \widehat{1}\}\right)$ associated to the dual poset $P^{*}$, obtained by reversing the partial order. Taking the dual changes the rank function, and thus the flag $f$-vector, but there is no change at the level of (nonflag) $f$-vectors. To simplify our notation and language we make the following definition.

Definition 3.1. Given a graded poset $P$, we call the Stirling vector, respectively Stirling polynomial, of $\triangle(P \backslash\{\widehat{0}, \widehat{1}\})$ the Stirling vector, respectively Stirling polynomial, of $P$. We use the notation $s_{P}(x)$ to denote the Stirling polynomial of $P$.

Our main result depends on the following lemma.

Lemma 3.2. Assume the numbers $c_{0}, \ldots, c_{n}$ satisfy

$$
c_{j}=\sum_{\substack{i, k \leq j \\
i+k \geq j}}\left(\begin{array}{c}
j \\
j-i, j-k, i+k-j
\end{array}\right) a_{i} b_{k},
$$


where $a_{0}, \ldots, a_{n}$ and $b_{0}, \ldots, b_{n}$ are given numbers. Then we have

$$
\sum_{j} c_{j}\left(\begin{array}{l}
x \\
j
\end{array}\right)=\sum_{i} a_{i}\left(\begin{array}{l}
x \\
i
\end{array}\right) \cdot \sum_{k} b_{k}\left(\begin{array}{l}
x \\
k
\end{array}\right) .
$$

Proof. Multiplying both sides with $\left(\begin{array}{l}x \\ j\end{array}\right)$ and summing over $j$ yields

$$
\sum_{j} c_{j}\left(\begin{array}{l}
x \\
j
\end{array}\right)=\sum_{j} \sum_{\substack{i, k \leq j \\
i+k \geq j}}\left(\begin{array}{c}
j \\
j-i, j-k, i+k-j
\end{array}\right) a_{i} b_{k}\left(\begin{array}{l}
x \\
j
\end{array}\right) .
$$

For a fixed pair $(i, k)$ on the right-hand side the product of the appropriate face numbers is multiplied with

$$
\sum_{j=\max (i, k)}^{i+k}\left(\begin{array}{c}
j \\
j-i, j-k, i+k-j
\end{array}\right)\left(\begin{array}{l}
x \\
j
\end{array}\right)
$$

which we claim to be equal to $\left(\begin{array}{l}x \\ i\end{array}\right) \cdot\left(\begin{array}{l}x \\ k\end{array}\right)$. In fact, without loss of generality we may assume that $x$ is a sufficiently large positive integer, since two polynomials from $\mathbb{R}[x]$ are equal, if they agree for infinitely many substitutions into $x$. Then the sum (3) counts the number of pairs of subsets $(Y, Z)$ of an $x$-element set $X$ such that $|Y|=i$ and $|Z|=k$ : introducing $j:=|Y \cup Z|$ there are $\left(\begin{array}{l}x \\ j\end{array}\right)$ ways to choose $Y \cup Z$, which then needs to be partitioned into a $(j-k)$-element set $Y \backslash Z$, a $(j-i)$-element set $Z \backslash Y$, and a $(k+i-j)$-element set $Y \cap Z$. On the other hand, we may choose $Y$ and $Z$ independently, giving $\left(\begin{array}{l}x \\ i\end{array}\right) \cdot\left(\begin{array}{l}x \\ k\end{array}\right)$ as stated. Therefore we may write

$$
\sum_{j} c_{j}\left(\begin{array}{l}
x \\
j
\end{array}\right)=\sum_{j} \sum_{\substack{i, k \leq j \\
i+k \geq j}} a_{i} b_{k}\left(\begin{array}{l}
x \\
i
\end{array}\right)\left(\begin{array}{l}
x \\
k
\end{array}\right)=\sum_{i} a_{i}\left(\begin{array}{l}
x \\
i
\end{array}\right) \cdot \sum_{k} b_{k}\left(\begin{array}{l}
x \\
k
\end{array}\right) .
$$

Our main result is the following.

Theorem 3.3. Any two graded posets $P$ and $Q$ satisfy

$$
s_{P \diamond Q}(x)=s_{P \diamond *}(x)=s_{P}(x) \cdot s_{Q}(x) .
$$

Proof. Let us compute the $f$-vector of $\triangle\left(P \diamond^{*} Q \backslash\{\widehat{0}, \widehat{1}\}\right)$ (the proof for the diamond product is identical). To obtain $f_{j-1}:=f_{j-1}\left(\triangle\left(P \diamond^{*} Q \backslash\{\widehat{0}, \widehat{1}\}\right)\right)$ we must enumerate all chains of the form $\widehat{0}<\left(p_{1}, q_{1}\right)<\cdots<\left(p_{j}, q_{j}\right)<\widehat{1}$ such that $\left\{p_{1}, \ldots, p_{j}\right\} \subseteq$ $P \backslash\left\{\widehat{1}_{P}\right\},\left\{q_{1}, \ldots, q_{j}\right\} \subseteq Q \backslash\left\{\widehat{1}_{Q}\right\}$, and $\left(p_{1}, q_{1}\right) \neq\left(\hat{0}_{P}, \widehat{0}_{Q}\right)=\widehat{0}$. Let us fix the sets $A:=\left\{p_{1}, \ldots, p_{j}\right\} \backslash\left\{\widehat{0}_{P}\right\}$ and $B:=\left\{q_{1}, \ldots, q_{j}\right\} \backslash\left\{\widehat{0}_{Q}\right\}$ and enumerate all chains for which $A$ and $B$ are the fixed sets. Starting from $\left(p_{0}, q_{0}\right):=\left(\widehat{0}_{P}, \widehat{0}_{Q}\right)$, each time we move one step up from $\left(p_{\alpha-1}, q_{\alpha-1}\right)$ to $\left(p_{\alpha}, q_{\alpha}\right)$ (where $\alpha=1,2, \ldots, j$ ) we must either change the first coordinate to the next larger element of $A$, or the second coordinate to the next larger element of $B$, or increase both coordinates to the next larger available element. Assuming $|A|=i$ and $|B|=k$, there must be exactly $j-k$ steps when only 
the first coordinate increases, $j-i$ steps when only the second coordinate increases, and $i+k-j$ steps when both coordinates increase. Thus we obtain

$$
f_{j-1}=\sum_{\substack{i, k \leq j \\
i+k \geq j}}\left(\begin{array}{c}
j \\
j-i, j-k, i+k-j
\end{array}\right) f_{i-1}(\triangle(P \backslash\{\widehat{0}, \widehat{1}\})) f_{k-1}(\triangle(Q \backslash\{\widehat{0}, \widehat{1}\}))
$$

and the statement follows from Lemma 3.2.

Remark 3.4. Any regular $C W$-complex $\Omega$ may be turned into a graded poset $P_{1}(\Omega)$ by adding a unique maximum element to the poset $P(\Omega)$ of its faces. (Posets of the form $P(\Omega)$ are described by Björner [5].) The order complex of $P_{1}(\Omega) \backslash\{\widehat{0}, \widehat{1}\}$ is identifiable with the barycentric subdivision of $\Omega$. Taking the dual diamond product of two such posets induces taking the free join of their order complexes, as mentioned in Corollary 1.3 of [15]. Hence the second sentence in the Abstract is a direct consequence of Theorem 3.3.

An important consequence of Theorem 3.3 is that the class of graded posets with nonnegative Stirling vectors is closed under taking the (dual) diamond product.

Corollary 3.5. If the Stirling vectors of the graded posets $P$ and $Q$ are non-negative then the Stirling vectors of $P \diamond Q$ and $P \diamond^{*} Q$ are also non-negative.

Repeated use of Theorem 3.3 yields the following.

Corollary 3.6. The Stirling polynomial of the face lattice of a d-dimensional cube is $(2 x+1)^{d}$.

Corollary 3.6 may be immediately generalized to the $r$-cubical lattice which may be defined as follows.

Definition 3.7. Let $\check{M}_{r}$ be the graded poset of rank 2 with $r$ atoms. The $r$-cubical lattice of rank $n+1$ is the $n$th diamond power of $\check{M}_{r}$.

Remark 3.8. In [9] Ehrenborg and Readdy use $M_{r}$ to denote $\check{M}_{r} \backslash\{\widehat{0}\}$, but they do not use yet the notion of the diamond product. The equivalence of their definition with the above one is straightforward. For further information and bibliography on $r$-cubical lattices, we refer the reader to their work.

Corollary 3.9. The Stirling polynomial of the r-cubical lattice of rank $n+1$ is $(r x+1)^{n}$.

\section{Graded Posets with Non-Negative Stirling Vectors}

In this section we present two large classes (and one "small" class) of graded posets consisting only of graded posets with non-negative Stirling vectors. We also provide 
examples of graded posets whose Stirling polynomial does have a negative coefficient. These examples help us avoid making false generalizations.

The first important class of graded posets with a non-negative Stirling vector is the class of simplicial posets, where a surprisingly simple connection exists.

Definition 4.1. A graded poset $P$ is simplicial if for every $x \in P \backslash \widehat{1}$ the interval $[\widehat{0}, x]$ is a Boolean algebra.

The surprising coincidence is that for simplicial posets the entries of the Stirling vector are not only positive but also have a very natural meaning.

Theorem 4.2. Every simplicial poset $P$ of rank $n+1$ satisfies

$$
s_{k}(\triangle(P \backslash\{\hat{0}, \widehat{1}\}))=f_{\{k\}}(P) \quad \text { for } k=1,2, \ldots, n \text { and } s_{0}(\triangle(P \backslash\{\widehat{0}, \widehat{1}\}))=1=f_{\emptyset}(P) \text {. }
$$

Here $f_{S}(P)$ is the appropriate entry from the flag $f$-vector.

Proof. For $j \geq 1$ we have

$$
f_{j-1}(\triangle(P \backslash\{\widehat{0}, \widehat{1}\}))=\sum_{k=j}^{n} f_{\{k\}}(P) \cdot S(k, j) \cdot j !
$$

In fact, when we choose a $j$-element chain $p_{1}<\cdots<p_{j}$ in $P \backslash\{0, \widehat{1}\}$, there are $f_{\{k\}}(P)$ ways to choose its top element $p_{j}$ at rank $k(k=j, j+1, \ldots, n)$ and then there are $S(k, j) \cdot j$ ! ways to choose a $(j-1)$-element chain in the open interval $\left(\widehat{0}, p_{j}\right)$. (Since $\left[\widehat{0}, p_{j}\right]$ is a Boolean algebra of rank $k$, choosing a $(j-1)$-element chain in $\left(\widehat{0}, p_{j}\right)$ is equivalent to choosing an ordered set-partition of a $k$-element set into $j$-parts.) Directly, we also have

$$
f_{-1}(\triangle(P \backslash\{\hat{0}, \widehat{1}\}))=1=f_{\emptyset}(P),
$$

thus the invertible matrix that sends the vector $\left(f_{\emptyset}(P), f_{\{1\}}(P), \ldots, f_{\{n\}}(P)\right)$ into the $f$ vector of $\triangle(P \backslash\{\hat{0}, \widehat{1}\})$ is the same as the matrix sending the Stirling vector of $\triangle(P \backslash\{\widehat{0}, \widehat{1}\})$ into its $f$-vector.

The next large class of posets is a subclass of graded planar posets.

Definition 4.3. A graded poset $P$ is planar if its Hasse diagram may be drawn in the plane with noncrossing edges, such that whenever $q \in P$ covers $p \in P$, the vertex representing $q$ is above the vertex representing $P$. We call a graded planar poset $P$ barely branching if for all $p \in P \backslash\{\widehat{0}, \widehat{1}\}$ at least one of the intervals $[\widehat{0}, p]$ and $[p, \widehat{1}]$ is a chain.

Example 4.4. A barely branching graded planar poset is shown in Fig. 1.

Theorem 4.5. The Stirling polynomial of a barely branching graded poset $P$ has nonnegative coefficients. 


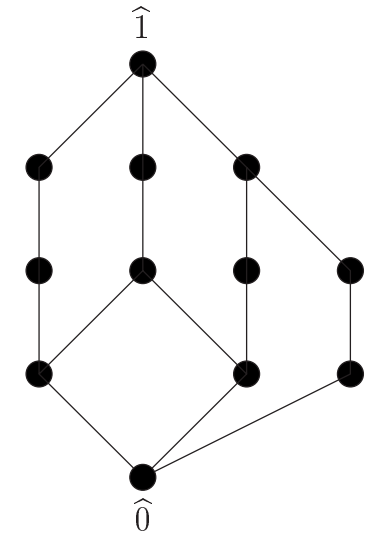

Fig. 1. A barely branching graded planar poset.

Proof. Assume $P$ has rank $n+1$, and let us draw in the plane and enumerate its saturated chains just like in [4]. This means that for each fixed $p \in P$ we number the edges $(p, q)$ in the Hasse diagram left to right, and then we enumerate the saturated chains in lexicographic order of the labels. It was shown in [4] that under these circumstances we may associate a set $S$ to each saturated chain $C$ in such a way that a subset $D$ of the chain $C$ appears first in $C$ if an only if the set of ranks $T$ of the $D$ has a nonempty intersection with every interval of $\mathcal{I}[S]$. Here $\mathcal{I}[S]$ is the unique family of intervals $\left\{\left[a_{1}, b_{1}\right], \ldots,\left[a_{k}, b_{k}\right]\right\}$ satisfying $S=\bigcup_{i=1}^{k}\left[a_{i}, b_{i}\right]$ and $a_{i+1} \geq b_{i}+2$ for $i \leq k-1$. The set $S$ associated to the saturated chain

$$
C: \widehat{0}=p_{0} \prec p_{1} \prec p_{2} \prec \cdots \prec p_{n} \prec p_{n+1}=\widehat{1}
$$

is determined as follows. Let $\mathcal{I}_{C}$ consist of all intervals $[u, v]$ for which there is a saturated chain $p_{u-1} \prec p_{u}^{\prime} \prec p_{u+1}^{\prime} \prec \cdots \prec p_{v}^{\prime} \prec p_{v+1}$ in $\left[p_{u-1}, p_{v+1}\right]$ such that the label of the cover relation $\left(p_{u-1}, p_{u}^{\prime}\right)$ is less than the label of $\left(p_{u-1}, p_{u}\right)$. (Equivalently, the saturated chain

$$
C^{\prime}: \widehat{0}=p_{0} \prec p_{1} \prec \cdots \prec p_{u-1} \prec p_{u}^{\prime} \prec \cdots p_{v}^{\prime} \prec p_{v} \prec \cdots p_{n} \prec p_{n+1}=\widehat{1}
$$

precedes $C$ in the lexicographic order.) By the results in [4] a subset of $C$ does not occur in any preceding saturated chain if and only if the set $T$ of ranks of its elements has a nonempty intersection with every element of $\mathcal{I}_{C}$. We get the same condition if we require only $T \cap I \neq \emptyset$ for the minimal intervals $I$ in $\mathcal{I}_{C}$. Finally, it was also shown in [4] that, for a graded planar poset, the family of minimal intervals of $\mathcal{I}_{C}$ is always of the form $\mathcal{I}[S]$ for some $S \subseteq\{1, \ldots, n\}$. (If the union of two minimal intervals of $\mathcal{I}_{C}$ is an interval then some edges are bound to cross in the Hasse diagram.)

We claim that the barely branching property implies that in the above enumeration every family of intervals $\mathcal{I}_{C}$ is either empty or has a unique minimum interval. In fact, if for some saturated chain

$$
C: \widehat{0}=p_{0} \prec p_{1} \prec p_{2} \prec \cdots \prec p_{n} \prec p_{n+1}=\widehat{1}
$$


the family $\mathcal{I}_{C}$ has at least two minimal intervals $\left[a_{1}, b_{1}\right]$ and $\left[a_{2}, b_{2}\right]$ then by what was said above we may assume $a_{2} \geq b_{1}+2$. Consider now the element $p:=p_{b_{1}+1}$ of rank $b_{1}+1$ in $C$. Since $\left[a_{1}, b_{1}\right] \in \mathcal{I}_{C}$ implies the existence of at least two saturated chains in $\left[p_{a_{1}-1}, p_{b_{1}+1}\right] \subseteq[\widehat{0}, p]$, and $\left[a_{2}, b_{2}\right] \in \mathcal{I}_{C}$ implies the existence of at least two saturated chains in $\left[p_{a_{2}-1}, p_{b_{2}+1}\right] \subseteq[p, \widehat{1}]$, the definition of a barely branching planar poset is violated by $p$.

It is thus sufficient to show that every saturated chain $C$, for which $\mathcal{I}_{C}$ is empty or has a unique minimal interval, increases the Stirling polynomial. Since for any graded poset $P$ we have

$$
f_{j-1}(\triangle(P \backslash\{\widehat{0}, \widehat{1}\}))=\sum_{\substack{T \subseteq[1, \ldots, n\} \\|T|=j}} f_{T}(P)
$$

in the case $\mathcal{I}_{C}=\emptyset$ we need to show that the polynomial

$$
\sum_{T \subseteq\{1, \ldots, n\}}\left(\begin{array}{c}
x \\
|T|
\end{array}\right)=\sum_{j=0}^{n}\left(\begin{array}{l}
n \\
j
\end{array}\right) \cdot\left(\begin{array}{l}
x \\
j
\end{array}\right)=\sum_{j=0}^{n}\left(\begin{array}{c}
n \\
n-j
\end{array}\right) \cdot\left(\begin{array}{c}
x \\
j
\end{array}\right)=\left(\begin{array}{c}
x+n \\
n
\end{array}\right)
$$

has non-negative coefficients. This is directly true. Similarly, in the case when the unique minimal interval of $\mathcal{I}_{C}$ is the interval $I$, we need to show that the polynomial

$$
\sum_{\substack{T \leq\{1, \ldots, n\} \\
T \cap I \neq \emptyset}}\left(\begin{array}{c}
x \\
|T|
\end{array}\right)
$$

has non-negative coefficients. Introducing $k:=|I|$ we may write

$$
\begin{aligned}
\sum_{\substack{T \subseteq 1, \ldots, n\} \\
T \cap \mid \neq \emptyset}}\left(\begin{array}{c}
x \\
|T|
\end{array}\right) & =\sum_{T \subseteq\{1, \ldots, n\}}\left(\begin{array}{c}
x \\
|T|
\end{array}\right)-\sum_{\substack{T \subseteq 1, \ldots, n\} \\
T \cap l=\emptyset}}\left(\begin{array}{c}
x \\
|T|
\end{array}\right)=\left(\begin{array}{c}
x+n \\
n
\end{array}\right)-\sum_{j=0}^{n-k}\left(\begin{array}{c}
n-k \\
j
\end{array}\right)\left(\begin{array}{c}
x \\
j
\end{array}\right) \\
& =\left(\begin{array}{c}
x+n \\
n
\end{array}\right)-\sum_{j=0}^{n-k}\left(\begin{array}{c}
n-k \\
n-k-j
\end{array}\right)\left(\begin{array}{c}
x \\
j
\end{array}\right)=\left(\begin{array}{c}
x+n \\
n
\end{array}\right)-\left(\begin{array}{c}
x+n-k \\
n-k
\end{array}\right) .
\end{aligned}
$$

Now our theorem follows from the fact that the polynomial

$$
\begin{aligned}
\left(\begin{array}{c}
x+n \\
n
\end{array}\right)-\left(\begin{array}{c}
x+n-k \\
n-k
\end{array}\right)= & \frac{(x+n-k) \cdots(x+1)}{(n-k) !} \\
& \cdot \frac{(x+n) \cdots(x+n-k+1)-n \cdots(n-k+1)}{n \cdots(n-k+1)}
\end{aligned}
$$

has non-negative coefficients. The subtracted product in the numerator of the second factor is the constant term of the first polynomial in the same numerator.

Remark 4.6. In [4] the number $p_{S}$ is introduced for the number of saturated chains satisfying $\mathcal{I}_{C}=\mathcal{I}[S]$. The resulting vector $\left(p_{S}: S \subseteq\{1, \ldots, n\}\right)$ is a planar analogue of the flag $h$-vector (whose definition may be also found in [4] but was widely used before). In terms of this flag $p$-vector the barely branching condition implies $p_{S}=0$ whenever $|\mathcal{I}[S]| \geq 2$. 
Remark 4.7. Consider an arbitrary graded planar poset of rank 4. The contribution of a saturated chain $C$ satisfying $\mathcal{I}_{C}=\mathcal{I}[\{1,3\}]$ to the Stirling polynomial is $\left(\begin{array}{l}x \\ 2\end{array}\right)+\left(\begin{array}{l}x \\ 3\end{array}\right)=$ $x^{3} / 6-x / 6$ since the only subsets of $\{1,2,3\}$ containing $\{1,3\}$ are $\{1,3\}$ itself and $\{1,2,3\}$. Then a sequence of planer posets $P_{N}:=P(3, \mathcal{I}[\{1,3\}], N)$, as defined in Definition 4 of [4], has the property

$$
\lim _{N \rightarrow \infty} \frac{1}{N^{2}} p_{S}\left(P_{N}\right)=\delta_{\{1,3\}, S},
$$

where $\delta_{\{1,3\}, S}$ is the Kronecker delta symbol. Thus, for a sufficiently large $N$, the coefficient of $x$ in the Stirling polynomial of the graded planar poset $P_{N}$ must be negative.

Our last example is a "smaller" class of graded posets, giving rise to a "false hope."

Proposition 4.8. The face lattice of any three-dimensional convex polytope has a nonnegative Stirling vector.

Proof. Using (4) we may rewrite the Stirling polynomial of an arbitrary graded poset $P$ of rank 4 in terms of its flag $f$-vector as follows:

$s_{P}(x)=1+\left(f_{\{1\}}+f_{\{2\}}+f_{\{3\}}\right) \cdot\left(\begin{array}{c}x \\ 1\end{array}\right)+\left(f_{\{1,2\}}+f_{\{2,3\}}+f_{\{1,3\}}\right) \cdot\left(\begin{array}{l}x \\ 2\end{array}\right)+f_{\{1,2,3\}} \cdot\left(\begin{array}{l}x \\ 3\end{array}\right)$.

The face lattice of a convex polytope is Eulerian, hence we may use the Bayer-Billera relations from Theorem 1.1, and replace each of $f_{\{1,2\}}, f_{\{2,3\}}$, and $f_{\{1,3\}}$ with $f_{\{1,2,3\}} / 2=$ $2 f_{\{2\}}$, the number $f_{\{1,2,3\}}$ with $4 f_{\{2\}}$, and the sum $f_{\{1\}}+f_{\{3\}}$ with $f_{\{2\}}+2$. Thus we obtain

$$
\begin{aligned}
s_{P}(x) & =1+\left(2 f_{\{2\}}+2\right) \cdot\left(\begin{array}{l}
x \\
1
\end{array}\right)+6 f_{\{2\}} \cdot\left(\begin{array}{l}
x \\
2
\end{array}\right)+4 f_{\{2\}} \cdot\left(\begin{array}{l}
x \\
3
\end{array}\right) \\
& =1+\left(\frac{1}{3} f_{\{2\}}+2\right) \cdot x+f_{\{2\}} \cdot x^{2}+\frac{4}{6} f_{\{2\}} \cdot x^{3},
\end{aligned}
$$

a polynomial with positive coefficients.

The "false hope" arising from Proposition 4.8 is that perhaps the face lattice of any convex polytope has a non-negative Stirling vector. This turns out to be false even for four-dimensional polytopes. In fact, for any Eulerian poset $P$ of rank 5, we may use (4) and the Bayer-Billera relations from Theorem 1.1 to express the $f$-vector $\Delta(P \backslash\{\widehat{0}, \widehat{1}\})$ in terms of the flag $f$-vector of $P$ as follows (to alleviate our notation, we write $f_{S}$ instead of $\left.f_{S}(P)\right)$ :

$$
\begin{aligned}
\left.f_{0}(\Delta(P \backslash \widehat{0}, \widehat{1}\})\right) & =f_{\{1\}}+f_{\{2\}}+f_{\{3\}}+f_{\{4\}} \\
& =2 f_{\{1\}}+2 f_{\{3\}}, \\
\left.f_{1}(\Delta(P \backslash \widehat{0}, \widehat{1}\})\right) & =f_{\{1,2\}}+f_{\{1,3\}}+f_{\{1,4\}}+f_{\{2,3\}}+f_{\{2,4\}}+f_{\{3,4\}} \\
& =f_{\{1,2\}}+f_{\{1,3\}}+\left(f_{\{1,3\}}-f_{\{1,2\}}+2 f_{\{1\}}\right)+f_{\{1,3\}}+2 f_{\{3\}} \\
& =2 f_{\{1\}}+2 f_{\{3\}}+3 f_{\{1,3\}},
\end{aligned}
$$




$$
\begin{aligned}
& f_{2}(\triangle(P \backslash\{\widehat{0}, \widehat{1}\}))=f_{\{1,2,3\}}+f_{\{1,2,4\}}+f_{\{1,3,4\}}+f_{\{2,3,4\}}=8 \cdot f_{\{1,3\}}, \\
& f_{3}(\triangle(P \backslash\{\widehat{0}, \widehat{1}\}))=f_{\{1,2,3,4\}}=4 \cdot f_{\{1,3\}} .
\end{aligned}
$$

As a consequence, the polynomial $s_{P}(x)$ may be written as

$$
\begin{aligned}
s_{P}(x)= & 1+\left(2 f_{\{1\}}+2 f_{\{3\}}\right) \cdot\left(\begin{array}{l}
x \\
1
\end{array}\right)+\left(2 f_{\{1\}}+2 f_{\{3\}}+3 f_{\{1,3\}}\right)\left(\begin{array}{l}
x \\
2
\end{array}\right) \\
& +8 \cdot f_{\{1,3\}}\left(\begin{array}{l}
x \\
3
\end{array}\right)+4 \cdot f_{\{1,3\}}\left(\begin{array}{l}
x \\
4
\end{array}\right) .
\end{aligned}
$$

Collecting terms yields

$$
\begin{aligned}
s_{P}(x)= & 1+\left(f_{\{1\}}+f_{\{3\}}+\frac{f_{\{1,3\}}}{6}\right) \cdot x+\left(f_{\{1\}}+f_{\{3\}}-\frac{2 \cdot f_{\{1,3\}}}{3}\right) \\
& \cdot x^{2}+\frac{f_{\{1,3\}}}{3} \cdot x^{3}+\frac{f_{\{1,3\}}}{6} \cdot x^{4} .
\end{aligned}
$$

Corollary 4.9. An Eulerian poset of rank 5 has a positive Stirling polynomial if and only if its flag $f$-vector satisfies $2 f_{\{1,3\}} \leq 3 f_{\{1\}}+3 f_{\{3\}}$.

If $P$ is the face lattice of a 4-polytope then $f_{\{1\}}$ stands here for the number of vertices (elements of rank 1), and $f_{\{3\}}$ for the number of 2 -faces. In most of the the literature on 4-polytopes the dimensions (rather than the ranks) of the faces are used to index the flag $f$-vector, so the condition of Corollary 4.9 would be written as

$$
2 f_{02} \leq 3 f_{0}+3 f_{2} \text {. }
$$

This inequality is not satisfied by all 4-polytopes, at least two known classes of counterexamples exist.

Example 4.10. In [26] Ziegler constructs polytopes $P(n, r)$ as projected products of polygons, whose flag $f$-vector (indexed by dimensions) satisfies

$$
\left(f_{0}, f_{1}, f_{2}, f_{3} ; f_{03}\right)=(4 n, 4 r n, 5 r n-6+4 r, r n-2+4 r ; 16 r n-16 n) \cdot \frac{1}{4} n^{r-1} .
$$

For large values of $n$ and $r$, the vector $\left(f_{0}, f_{1}, f_{2}, f_{3} ; f_{03}\right)$ is approximately equal to $(0,4,5,1 ; 16) \cdot r n^{r} / 4$, and so we have

$$
f_{02}=f_{03}+2 f_{1}-2 f_{0} \sim(16+2 \cdot 4-2 \cdot 0) \cdot \frac{r n^{r}}{4}=24 \cdot \frac{r n^{r}}{4}
$$

while $3 f_{0}+3 f_{2}$ is only about $15\left(r n^{r} / 4\right)$.

The other set of counterexamples consists of cubical polytopes, as discussed in the next section, hence there is no cubical analogue of Theorem 4.2.

It is worth noting that, although general Stirling positivity fails for four-dimensional polytopes, there seems to be no easier way to find a counterexample than using some of 
the most recent and highly nontrivial constructions. The neighborly cubical polytopes mentioned in Example 5.2 actually disproved a conjecture on the face numbers of a cubical polytope, while the polytopes mentioned in Example 4.10 were constructed with the purpose to get close to the suspected boundary of the cone of flag $f$-vectors of 4polytopes. The class of polytopes with non-negative Stirling polynomials is reasonably large, by virtue of the following.

Corollary 4.11. The face lattice of a simplicial or simple polytope has a non-negative Stirling vector.

Corollary 4.11 is a consequence of Theorem 4.2 and the invariance under taking the dual noted at the beginning of Section 3, which has the following implication for polytopes.

Corollary 4.12. The class of polytopes having a non-negative Stirling vector is closed under taking the dual of a polytope.

As underscored by Ehrenborg and Fox [8], taking the Cartesian product of two polytopes induces taking the diamond product of their face lattices. (At this point, Ehrenborg and Fox cite Kalai [17] who uses the term lower truncated product instead of diamond product and upper truncated product instead of the dual diamond product.) Thus, Theorem 3.3 has the following corollary.

Corollary 4.13. The class of polytopes having a non-negative Stirling vector is closed under taking the Cartesian product.

\section{Cubical Posets and an Identity for Bernoulli Polynomials}

Definition 5.1. A graded poset $P$ is a cubical poset if for every $p \in P \backslash \widehat{\{1}\}$ the interval $[\widehat{0}, p]$ is isomorphic to the face lattice of a cube. A convex polytope is cubical if its face lattice is a cubical poset.

Note that cubical polytopes satisfy $f_{02}=4 f_{2}$ and so (5) is equivalent to

$$
f_{2} \leq 3 f_{0} .
$$

Example 5.2. Joswig and Ziegler have shown [16] that neighborly cubical polytopes exist: for any $n \geq d \geq 2 r+2$ there is a cubical convex $d$-polytope, whose $r$-skeleton is combinatorially equivalent to that of the $n$-dimensional cube. For $d=4$ the neighborly cubical polytopes exhibit the fact that the facet-vertex ratio $f_{3} / f_{0}$ is not bounded. Using the Bayer-Billera relations from Theorem 1.1 we may rewrite (6) as $f_{1}+f_{3}-f_{0} \leq 3 f_{0}$ or, equivalently,

$$
f_{1} / f_{0}+f_{3} / f_{0} \leq 4
$$

This inequality is violated by any neighborly cubical polytope satisfying $f_{3} / f_{0}>4$. 
Even if the Stirling polynomial of a cubical poset does not necessarily have non-negative coefficients, it is worth noting that computing the Stirling polynomial of a cubical poset may be achieved by using a more general method, which may serve in the future as a tool to prove the non-negativity of the Stirling vector of some other classes of graded posets. This method consists of calculating the Stirling vector of an arbitrary graded poset $P$ from the Stirling vectors of some of its proper intervals.

Lemma 5.3. The Stirling polynomial $s_{P}(x)$ of any graded poset $P$ satisfies

$$
s_{P}(x+1)-s_{P}(x)=\sum_{p \in P \backslash\{\hat{0}, \hat{1}\}} s_{[\hat{0}, p]}(x) .
$$

Proof. As noted in the proof of Theorem 4.2, the $j$-element chains in $P \backslash\{\widehat{0}, \widehat{1}\}$ may be enumerated by first choosing the top element $p$ of the chain, and then choosing a $(j-1)$-element chain in the open interval $(\widehat{0}, p)$. Thus we have

$$
s_{P}(x)=1+\sum_{j=1}^{n} \sum_{p \in P \backslash\{\hat{0}, \hat{1}\}} f_{j-2}(\triangle(\widehat{0}, p)) \cdot\left(\begin{array}{l}
x \\
j
\end{array}\right) .
$$

Substituting $x+1$ for $x$ in the above equation and taking the difference yields

$$
s_{P}(x+1)-s_{P}(x)=\sum_{j=1}^{n} \sum_{p \in P \backslash\{\hat{0}, \hat{1}\}} f_{j-2}(\triangle(\widehat{0}, p)) \cdot\left(\left(\begin{array}{c}
x+1 \\
j
\end{array}\right)-\left(\begin{array}{c}
x \\
j
\end{array}\right)\right) .
$$

As a consequence of the well-known identity

$$
\left(\begin{array}{c}
x+1 \\
j
\end{array}\right)-\left(\begin{array}{c}
x \\
j
\end{array}\right)=\left(\begin{array}{c}
x \\
j-1
\end{array}\right)
$$

the polynomial associated to each $p$ is the Stirling polynomial of $[\widehat{0}, p]$.

Corollary 5.4. For any positive integer $x$ we have

$$
s_{P}(x)=1+\sum_{p \in P \backslash\{\hat{0}, \hat{1}\}} \sum_{y=0}^{x-1} s_{[\hat{0}, p]}(y) .
$$

In fact, we have $s_{P}(x)=s_{P}(0)+\sum_{y=0}^{x-1}\left(s_{P}(y+1)-s_{P}(y)\right)$, so the corollary follows from Lemma 5.3 and the constant term $s_{P}(0)=1$.

Corollary 5.4 is especially useful when we consider a class of posets for which every interval of the form $[\widehat{0}, p]$ belongs to a smaller class, as is the case for simplicial or cubical posets. If this smaller class is selected in such a way that even the polynomial $\sum_{y=0}^{x-1} s_{[0, p]}(y)$ has non-negative coefficients then the Stirling polynomial of the entire poset also has non-negative coefficients. (The use of the word "even" is justified as follows. If $\psi(x)$ is a polynomial then $\varphi(x)=\sum_{y=0}^{x-1} \psi(y)$ is a polynomial function, determined up to its constant term. The polynomial $\varphi(x)$ may be equivalently defined 
by the equation $\varphi(x+1)-\varphi(x)=\psi(x)$. Clearly, if $\varphi(x)$ has non-negative coefficients, the same holds for $\psi(x)$.) This stronger non-negativity is true for Boolean algebras, but false for the face lattices of cubes.

As a consequence of Corollaries 3.6 and 5.4 we obtain the following formula for the Stirling polynomial of a cubical poset $P$ of rank $n+1$. For a positive integer $x$, we must have

$$
s_{P}(x)=1+\sum_{k=1}^{n} f_{\{k\}} \cdot \sum_{y=0}^{x-1}(2 y+1)^{k-1}
$$

More generally, if in a graded poset $P$ every interval $[\widehat{0}, x](x<\widehat{1})$ is an $r$-cubical lattice, we have

$$
s_{P}(x)=1+\sum_{k=1}^{n} f_{\{k\}} \cdot \sum_{y=0}^{x-1}(r y+1)^{k-1} .
$$

This formula may be simplified using Bernoulli polynomials.

Definition 5.5. The Bernoulli polynomials $B_{n}(x)(n=0,1, \ldots)$ are given by the generating function

$$
\frac{t \cdot e^{x t}}{e^{t}-1}=\sum_{n=0}^{\infty} B_{n}(x) \cdot \frac{t^{n}}{n !}
$$

It is well known that the Bernoulli polynomials may be used to express the sum of fixed powers of consecutive integers. For any positive integer $m$ and $n$ we have

$$
\sum_{k=1}^{m} k^{n}=\frac{B_{n+1}(m+1)-B_{n+1}(0)}{n+1} .
$$

See Sections 23.1.2 and 23.1.4 of [1]. Hence it is not surprising that Bernoulli polynomials may be also used to express the sum of fixed powers of integers from any arithmetic sequence.

Lemma 5.6. For any positive integer $x$ and $k$ we have

$$
\sum_{y=0}^{x-1}(r y+1)^{k-1}=\frac{r^{k-1}}{k} \cdot\left(B_{k}\left(x+\frac{1}{r}\right)-B_{k}\left(\frac{1}{r}\right)\right) .
$$

This lemma is easy to derive directly, and it is also a special case of the following more general formula of Dattoli et al. [7, Formula (2.3)]:

$$
\sum_{n=0}^{N-1}(x+n y)^{r}=\frac{y^{r}}{r+1} \cdot\left(B_{r+1}\left(N+\frac{x}{y}\right)-B_{r+1}\left(\frac{x}{y}\right)\right) \quad \text { if } \quad y \neq 0 .
$$

Using Lemma 5.6 we may rewrite (7) in a more compact form and summarize our findings in the following theorem. 
Theorem 5.7. If in a graded poset $P$ every interval $[\widehat{0}, x]$ satisfying $x<\widehat{1}$ is an $r$-cubical lattice, then we have

$$
s_{P}(x)=1+\sum_{k=1}^{n} f_{\{k\}} \cdot \frac{r^{k-1}}{k} \cdot\left(B_{k}\left(x+\frac{1}{r}\right)-B_{k}\left(\frac{1}{r}\right)\right) .
$$

In particular, the Stirling polynomial of a cubical poset of rank $n+1$ is given by

$$
s_{P}(x)=1+\sum_{k=1}^{n} f_{\{k\}} \cdot \frac{2^{k-1}}{k} \cdot\left(B_{k}\left(x+\frac{1}{2}\right)-B_{k}\left(\frac{1}{2}\right)\right) .
$$

Note that (7) was only stated for positive integer values of $x$ while in Theorem 5.7 we do not make such restriction. As usual, this may be justified by the fact that two polynomials from $\mathbb{R}[x]$ are equal if they yield the same value for infinitely many substitutions.

Theorem 5.7 provides a second alternative to compute the Stirling polynomial of the $r$-cubical lattice. Thus, together with Corollary 3.9, it yields the following identity for Bernoulli polynomials:

Corollary 5.8. The Bernoulli polynomials satisfy

$$
1+r^{n} \sum_{k=1}^{n} \frac{\left(\begin{array}{c}
n \\
k-1
\end{array}\right)}{k} \cdot\left(B_{k}\left(x+\frac{1}{r}\right)-B_{k}\left(\frac{1}{r}\right)\right)=(r x+1)^{n} \quad \text { for all } \quad n \geq 0 .
$$

In fact, the number of elements of rank $k$ in an $r$-cubical lattice of rank $n+1$ is $f_{\{k\}}=$ $\left(\begin{array}{c}n \\ k-1\end{array}\right) \cdot r^{n+1-k}$, and multiplying this number by $r^{k-1} / k$ yields $\left(\begin{array}{c}n \\ k-1\end{array}\right) \cdot r^{n} / k$.

\section{Connection to the Gessel-Stanley Stirling Polynomials}

The term "Stirling polynomial" is already used in more than one sense in the literature. In this section we show that there exists a simplicial complex whose Stirling polynomial is closely related to the Stirling polynomial $f_{n}(m)=S(n+m, m)$ introduced by Gessel and Stanley in [14]. (See also Supplementary Problem 3.2 of [25].)

Definition 6.1. Given a finite set $X$, we define the cladistic complex $\mathcal{C}(X)$ on $X$ to be the following simplicial complex:

(i) Its vertices are the proper subsets $X$.

(ii) Its nonempty faces are all families $\mathcal{F}$ of proper subsets of $X$ such that for any $A, B \in \mathcal{F}$ either $A \cap B=\emptyset$, or $A \subseteq B$, or $B \subseteq A$ holds.

It is easy to verify that $\mathcal{C}(X)$ is in deed a simplicial complex. If we add to each family $\mathcal{F} \in \mathcal{C}(X)$ the set $X$ itself, we obtain the cladistic characters or trees of subsets of $X$ studied in the work of McMorris and Zaslavsky [21]. They show that the number $t(n, r)$ of cladistic characters of $[n]=\{1,2, \ldots, n\}$ with $r$ elements satisfies the recursion formula

$$
t(n, r)=r t(n-1, r)+2(r-1) t(n-1, r-1)+(r-2) t(n-1, r-2) .
$$


Introducing $f_{j-1}^{n}$ as a shorthand for $f_{j-1}(\mathcal{C}([n]))$, and using $f_{j-1}^{n}=t(n, j+1)$, we may rewrite (10) as

$$
f_{j-1}^{n}=(j+1) \cdot f_{j-1}^{n-1}+2 j \cdot f_{j-2}^{n-1}+(j-1) \cdot f_{j-3}^{n-1} .
$$

To make this formula valid even for "small" values of $j$, we use the convention $f_{k}^{n}=0$ for $k<-1$.

Theorem 6.2. The Stirling polynomial $s_{\mathcal{C}([n])}(m)$ of the cladistic complex $\mathcal{C}([n])$ is $S(n+m, m+1)$.

Proof. On the one hand,

$$
\begin{aligned}
s_{\mathcal{C}([n])}(m)-s_{\mathcal{C}([n])}(m-1) & =\sum_{j \geq 0} f_{j-1}^{n}\left(\left(\begin{array}{c}
m \\
j
\end{array}\right)-\left(\begin{array}{c}
m-1 \\
j
\end{array}\right)\right)=\sum_{j \geq 1} f_{j-1}^{n}\left(\begin{array}{c}
m-1 \\
j-1
\end{array}\right) \\
& =\sum_{k \geq 0} f_{k}^{n}\left(\begin{array}{c}
m-1 \\
k
\end{array}\right) .
\end{aligned}
$$

On the other hand,

$$
(m+1) \cdot s_{\mathcal{C}[n-1]}(m)=\sum_{j \geq 0} f_{j-1}^{n-1}(m+1)\left(\begin{array}{c}
m \\
j
\end{array}\right)=\sum_{j \geq 0} f_{j-1}^{n-1}(j+1)\left(\begin{array}{c}
m+1 \\
j+1
\end{array}\right) .
$$

Since

$$
\left(\begin{array}{c}
m+1 \\
j+1
\end{array}\right)=\left(\begin{array}{c}
m \\
j
\end{array}\right)+\left(\begin{array}{c}
m \\
j+1
\end{array}\right)=\left(\begin{array}{c}
m-1 \\
j-1
\end{array}\right)+2\left(\begin{array}{c}
m-1 \\
j
\end{array}\right)+\left(\begin{array}{c}
m-1 \\
j+1
\end{array}\right)
$$

we may rewrite (13) as

$$
(m+1) \cdot s_{\mathcal{C}[n-1]}(m)=\sum_{k \geq 0}\left(f_{k}^{n-1} \cdot(k+2)+f_{k-1}^{n-1} \cdot 2(k+1)+f_{k-2}^{n-1} \cdot k\right)\left(\begin{array}{c}
m-1 \\
k
\end{array}\right) .
$$

As an immediate consequence of (11), the right-hand side of (12) is the same as the right-hand side of (14). Thus the entries in the array $\left(s_{\mathcal{C}([n])}(m)\right)_{n, m \geq 0}$ satisfy the same recursion formula as those in the array of Stirling numbers $(S(n+m, m+1))_{n, m \geq 0}$, for which

$$
S(n+m, m+1)-S(n-1+m, m)=(m+1) S(n-1+m, m+1)
$$

holds. Therefore we may proceed by induction on $m$, keeping in mind that for all $n \geq 0$ we have $s_{\mathcal{C}([n])}(0)=S(n, 1)=1$ and $s_{\mathcal{C}([n])}(1)=1+f_{0}^{n}=1+t(n, 2)=2^{n}-1=$ $S(n+1,2)$. 


\section{Concluding Remarks}

Reasons to continue exploring the Stirling polynomials of face lattices of polytopes are presented at the end of Section 4. Corollary 5.4, together with (9) indicates that Bernoulli polynomials are likely to play an important role in Stirling polynomial calculations of graded posets, not only in the cubical case.

We remind the reader that another way to calculate the sum of powers $1^{r}+\cdots+n^{r}$ is by means of the Eulerian number $A_{r, i}$ expressing the number of permutations of $r$ numbers with $i$ descents. The formula may be found by multiplying both sides of the well-known identity

$$
\sum_{k=0}^{\infty} k^{r} \cdot t^{k}=\frac{\sum_{i=0}^{r-1} A_{r, i} t^{i+1}}{(1-t)^{r+1}}
$$

(see, e.g., Section 6.5 of [6]) by $(1-t)^{-1}$. This approach may also yield some interesting formulas for cubical posets, since the Eulerian numbers have an interpretation as volumes of certain slices of a hypercube (see [22] and the generalization by Ehrenborg et al. [13]). Another interesting approach to Eulerian numbers may be found in the recent work of Lam and Postnikov [19]. Since even among cubical polytopes some do not have a nonnegative Stirling vector, one would need to find a special property that would guarantee Stirling positivity and see whether in that special setting the use of Eulerian numbers and their interpretations as volumes is helpful. It is also worth exploring whether the Stirling vector of a zonotope would always be non-negative. We leave these questions as challenges to the reader.

\section{Acknowledgments}

I thank Louis Billera, Anders Björner, and Günter Ziegler for valuable advice and information on 4-polytopes. Thomas Zaslavsky helped me understand the structure of cladistic characters, and both my referees have greatly improved the quality of this work. In particular, one of my referees suggested all $r$-cubical generalizations of my formulas which were stated for cubical posets only.

\section{References}

1. M. Abramowitz and I. A. Stegun, Handbook of Mathematical Functions, National Bureau of Standards, Washington, DC, issued 1964, tenth printing, 1972, with corrections.

2. M. M. Bayer and L. J. Billera, Generalized Dehn-Sommerville relations for polytopes, spheres and Eulerian partially ordered sets, Invent. Math. 79 (1985), 143-157.

3. M. Bayer and A. Klapper, A new index for polytopes, Discrete Comput. Geom. 6 (1991), 33-47.

4. L. J. Billera and G. Hetyei, Decompositions of partially ordered sets, Order 17 (2000), 141-166.

5. A. Björner, Posets, regular CW-complexes and Bruhat order, European J. Combin. 5 (1984), 7-16.

6. L. Comtet, Advanced Combinatorics, Reidel, Dodrecht, 1974.

7. G. Dattoli, S. Lorenzutta, and C. Cesarano, Finite sum and generalized forms of Bernoulli polynomials, Rend. Mat. Appl. (7) 19 (1999), 385-391.

8. R. Ehrenborg and H. Fox, Inequalities for $c d$-indices of joins and products of polytopes, Combinatorica 23 (2003), 427-452. 
9. R. Ehrenborg and M. Readdy, The $r$-cubical lattice and a generalization of the $c d$-index, European $J$. Combin. 17 (1996), 709-725.

10. R. Ehrenborg and M. Readdy, Coproducts and the $c d$-index, J. Algebraic Combin. 8 (1998), 273-299.

11. R. Ehrenborg and M. Readdy, Homology of Newtonian coalgebras, European J. Combin. 23 (2002), 919-927.

12. R. Ehrenborg and M. Readdy, The Tchebyshev transforms of the first and second kind, Preprint, 2004, arXiv:math.CO/0412124.

13. R. Ehrenborg, M. Readdy, and E. Steingrímsson, Mixed volumes and slices of the cube, J. Combin. Theory Ser. A 81 (1998), 121-126.

14. I. Gessel and R. Stanley, Stirling polynomials, J. Combin. Theory Ser. A 24 (1978), 24-33.

15. G. Hetyei, Polyspherical complexes, Preprint, 2004, to appear in the Annals of Combinatorics.

16. M. Joswig and G. M. Ziegler, Neighborly Cubical Polytopes, Discrete Comput. Geom. 24 (2000), 325-444.

17. G. Kalai, A new basis for polytopes, J. Combin. Theory Ser. A 49 (1988), 191-209.

18. K. Karu, The $c d$-index of fans and lattices, Preprint, 2004, arXiv:math.AG/0410513.

19. T. Lam and A. Postnikov, Alcoved polytopes, Preprint, 2005, arXiv:math.CO/0501246.

20. J. P. May, A Concise Course in Algebraic Topology, The University of Chicago Press, Chicago, 1999.

21. F. R. McMorris and T. Zaslavsky, The number of cladistic characters, Math. Biosci. 54 (1981), 3-10.

22. R. P. Stanley, Eulerian partitions of a unit hypercube, in: Higher Combinatorics, Proceedings of the NATO Advanced Study Institute, Berlin, West-Germany, September 1-10, 1976 (M. Aigner, ed.), p. 49, Reidel, Dordrecht, 1977.

23. R. P. Stanley, Flag $f$-vectors and the $c d$-index, Math. Z. 216 (1994), 483-499.

24. R. P. Stanley, Combinatorics and Commutative Algebra, second edn., Birkhäuser, Boston, MA, 1996.

25. R. P. Stanley, Enumerative Combinatorics, Volume I, Cambridge University Press, Cambridge, 1997.

26. G. M. Ziegler, Projected products of polygons, Electron. Res. Announc. Amer. Math. Soc. 10 (2004), $122-134$.

Received December 17, 2004, and in revised form May 3, 2005. Online publication September 19, 2005. 